\title{
Correspondence
}

\section{Predicted values: how should we use them?}

Your editorial by Drs M R Miller and A C Pincock (April $1988 ; 43: 265-7)$ advocates reporting lung function in standard deviation units. This approach is statistically valid when the subject whose results are under scrutiny is selected at random. Oldham, however, has pointed out that people who become patients often do so because they are at increased risk; in these circumstances there is no compelling logic in the use of standard deviation units. ${ }^{1}$ Their use is implicit in the definition of normal lung function adopted by the European Society for Clinical Respiratory Physiology ${ }^{2}$ and formerly by the American Thoracic Society (ATS). But the ATS has now reverted to defining a normal result as one which is not more than $20 \%$ below the reference value.

The use of percentages has the apparent advantage that loss of lung function can be readily classified as mild, moderate, or severe, in the case of $F E V_{\text {, with cut off points at }}$ $80 \%, 60 \%$, and $40 \%$ of the reference value. These grades are then equated with loss of exercise capacity. ${ }^{3}$ Unfortunately, extrapolation from lung function to disability is not supported by evidence, ${ }^{4}$ and use of SD units does not overcome the difficulty. ${ }^{5}$

That the extrapolation is made at all indicates a need to consider how lung function results should be used and how reference values should be chosen for different applications. Your editorial only starts to address this issue and more needs to be done.

JE COTES

Department of Occupational Health and Hygiene Medical School Newcastle upon Tyne

1 Oldham PD. Percent of predicted as the limit of normal in pulmonary function testing: a stastistically valid approach. Thorax 1979;34:569-70.

2 De Coster A. Respiratory impairment and disablement. Bull Eur Physiopathol Respir 1983;19:1-3P.

3 American Thoracic Society. Evaluation of impairment/disability secondary to respiratory disorders. Am Rev Respir Dis 1986;133:1205-9.
4 Cotes JE, Zejda J, King B. Lung function impairment as guide to exercise limitation in work-related lung disorders. $A m \operatorname{Rev} \overrightarrow{\overline{\vec{B}}}$ Respir Dis 1988;137:1089-93.

5 Kauffmann F, Drouet D. Spirographic reference values-standardisation for what? Am Rev Respir Dis 1982;126:595.

AUTHORS' REPLY Dr Cotes holds the belief, previouslyळ

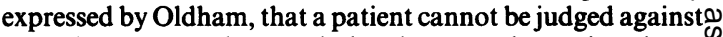
a random "normal" population because the patient is no longer a random subject from that population. If an inves-. tigator believes this to be true then any method of relating to a predicted value is invalid as the investigator believes that $a \omega$ predicted value based on a "normal" population has no meaning. The argument about demographic or other $\vec{x}$ differences in population questions whether any prediction can be made and does not have any bearing on the method of $\omega$ comparison. Many workers believe that a prediction can bein made from "normal" population data because most patients were originally part of the random population. It can bed argued that patients with congenital abnormalities of the윽 thorax, for instance, have never been part of any random "normal" population. Having made the assumptions neces- $z$ sary to make a prediction of lung function for a patient, we

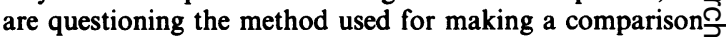
with this predicted value.

Dr Cotes states that $80 \%, 60 \%$, and $40 \%$ of predicted for $\mathrm{FEV}_{1}$ readily lead to classification in terms of mild, moderate, 6 and severe loss of lung function. As we have stated previously, this convenient statement ignores the fact that, in population terms, a fixed percentage of the predicted value represents a larger deviation from predicted values for youngo subjects than for old. Hence this convenient classification $\frac{0}{\mathbb{D}}$ introduces an age bias. Also any arbitrary classification such as this cannot necessarily be used for any other index.

While we disagree with Dr Cotes's statements as indicated 3 above, our respective concluding remarks are in agreement in that these issues need to be further addressed.

MR MILLER-

University of Birmingham Department of Medicine Good Hope District General Hospitak Sutton Coldfield, West Midlands. AC PINCOCK

University of Birmingham Department of Medicine. Queen Elizabeth Hospitab Birmingham 